\title{
Correlations and scaling properties of nonequilibrium fluctuations in liquid mixtures
}

\author{
Doriano Brogioli, ${ }^{1}$ Fabrizio Croccolo, ${ }^{2,3}$ and Alberto Vailati ${ }^{4}$ \\ ${ }^{1}$ Energiespeicher- und Energiewandlersysteme, Universität Bremen, Wiener Straße 12, 28359 Bremen, Germany \\ ${ }^{2}$ Laboratoire des Fluides Complexes et leurs Réservoirs-CNRS UMR5150, Université de Pau et des Pays de l'Adour, 64600 Anglet, France \\ ${ }^{3}$ Centre National d'Etudes Spatiales (CNES), 2, Place Maurice Quentin, 75001 Paris, France \\ ${ }^{4}$ Dipartimento di Fisica, Università degli Studi di Milano, I-20133 Milano, Italy
}

(Received 22 June 2016; published 29 August 2016)

\begin{abstract}
Diffusion in liquids is accompanied by nonequilibrium concentration fluctuations spanning all the length scales comprised between the microscopic scale $a$ and the macroscopic size of the system, $L$. Up to now, theoretical and experimental investigations of nonequilibrium fluctuations have focused mostly on determining their mean-square amplitude as a function of the wave vector. In this work, we investigate the local properties of nonequilibrium fluctuations arising during a stationary diffusion process occurring in a binary liquid mixture in the presence of a uniform concentration gradient, $\nabla c_{0}$. We characterize the fluctuations by evaluating statistical features of the system, including the mean-square amplitude of fluctuations and the corrugation of the isoconcentration surfaces; we show that they depend on a single mesoscopic length scale $l=\sqrt{a L}$ representing the geometric average between the microscopic and macroscopic length scales. We find that the amplitude of the fluctuations is very small in practical cases and vanishes when the macroscopic length scale increases. The isoconcentration surfaces, or fronts of diffusion, have a self-affine structure with corrugation exponent $H=1 / 2$. Ideally, the local fractal dimension of the fronts of diffusion would be $D_{l}=d-H$, where $d$ is the dimensionality of the space, while the global fractal dimension would be $D_{g}=d-1$. The transition between the local and global regimes occurs at a crossover length scale of the order of the microscopic length scale $a$. Therefore, notwithstanding the fact that the fronts of diffusion are corrugated, they appear flat at all the length scales probed by experiments, and they do not exhibit a fractal structure.
\end{abstract}

DOI: 10.1103/PhysRevE.94.022142

\section{INTRODUCTION}

Diffusion in liquids is a fundamental mass transfer process arising in the presence of a concentration gradient. When two miscible liquids are brought into contact, the random thermal motion of their molecules determines a diffusion process, which eventually gives rise to their uniform mixing at the molecular level. Even for a mixture of liquids at equilibrium, where the concentration is assumed to be uniform, the thermal agitation of the molecules gives rise to concentration fluctuations with a white noise spectrum, which relax back to the average concentration by diffusion. In the presence of a macroscopic concentration gradient, the amplitude of concentration fluctuations becomes orders of magnitude larger than the equilibrium one. These nonequilibrium fluctuations originate from the thermally excited velocity fluctuations that displace parcels of fluid in layers with different concentration. This mechanism was first predicted for binary mixtures in the late 1980s by Law and Nieuwoudt [1,2], who showed that a stationary diffusive process induced by a temperature gradient through the Soret effect is accompanied by concentration fluctuations exhibiting long-range correlations characterized by a power-law divergence $q^{-4}$ of their mean-square amplitude as a function of wave vector $q$. The power-law behavior is associated with a generic scale invariance of the fluctuations [3], in contrast with the critical scale invariance generated by the proximity to a critical point.

This finding was confirmed experimentally by a series of thorough experiments performed by Sengers et al. using dynamic light scattering [4-6]. Further theoretical [7] and experimental [8] investigation showed that on Earth, the scale invariance of the fluctuations is broken by the force of gravity, which stabilizes long-wavelength fluctuations and thus prevents their divergence. The same gravitational stabilization of the fluctuations was shown to be present during timedependent isothermal diffusion processes [9-12], proving that nonequilibrium fluctuations are a general feature of diffusive processes, irrespective of the origin of the concentration gradient driving them. An additional mechanism breaking the scale invariance of the fluctuations at small wave vectors was predicted theoretically to be the finite size of the sample [13], a finding confirmed experimentally during the GRADFLEX experiment by the European Space Agency [14-16]. Recent experiments showed that finite-size effects also affect the dynamics of the fluctuations in the presence of gravity [17]. The current understanding of nonequilibrium fluctuations is that they do not represent a mere perturbation of macroscopic nonequilibrium. Indeed, theoretical calculations and simulations have shown that macroscopic diffusive flow is generated entirely by the advective contribution of nonequilibrium fluctuations [18-20]. Therefore, the Fickean flux can be decomposed into a superposition of the microscopic currents generated by nonequilibrium fluctuations. A very recent advancement in the understanding of nonequilibrium fluctuations has been the theoretical prediction that long-range nonequilibrium fluctuations occurring in diffusive processes give rise to giant nonequilibrium Casimir forces that are orders of magnitude larger than those present in a mixture close to its critical point [21-23]. Earlier studies of nonequilibrium fluctuations relied on the investigation of their average static and dynamic structure factors, due to the fact that these quantities can be accessed easily by using optical techniques such as small-angle light scattering and a quantitative shadowgraph. However, 
recent studies on Casimir forces have outlined the need for a deeper understanding of the local properties of the fluctuations. In experiments with diffusing fluids performed by using small-angle light scattering and quantitative near-field techniques, designed for capturing images of the fluctuations, the amplitude of nonequilibrium fluctuations appears to be several orders of magnitude larger than that of equilibrium fluctuations at small wave vectors. One could then argue whether these giant nonequilibrium fluctuations could affect processes such as chemical reactions or the growth of crystals, whose kinetics are strongly influenced by the local concentration. The solution of this problem is not easy to achieve experimentally, due to the difficulty of probing the fluctuations locally. For example, a shadowgraph can achieve incredibly high interferometric sensitivities that allow us to map phase modulations much smaller than $2 \pi$, but the imaged quantity is not representative of the local concentration but rather of the whole optical path of light rays integrated across the sample thickness.

In this work, we characterize the fluctuations by evaluating statistical properties that have not been reported up to now. Such properties include the average amplitude and the correlation properties of the fluctuations along the concentration gradient and perpendicular to it. Moreover, we investigate the structure of the fronts of diffusion, which are corrugated due to the presence of the nonequilibrium fluctuations. We show that under realistic experimental conditions, the amplitude of concentration fluctuations is small compared to the macroscopic concentration differences that generate them, the ratio being of the order of $1 / 10^{4}$ if the gradient extends over lengths of the order of millimeters. For this reason, we argue that they are not likely to give rise to measurable effects in chemical reactions or in the growth of crystals (although the macroscopic concentration gradient itself can affect the reaction-diffusion behavior of the system [24,25]).

The power spectrum of the fluctuations is characterized by a power-law dependence on the wave vector, on length scales up to a lower cutoff depending on the system. In the presence of gravity, the cutoff is of the order of a fraction of a millimeter [8]; microgravity experiments [14,16] extended this cutoff by roughly a factor 30 . The power-law dependence led to the conclusion that the fluctuations are self-similar in a wide range of wave vectors, and to the argument that the fronts of diffusion are fractal (see, for example, the discussion in [26] and references therein). The analysis of experimental results obtained in microgravity confirmed the power-law behavior of the static structure factor of the fluctuations over a wide range of wave vectors, but it was not able to provide further insights about the fractal structure of the fronts of diffusion [14-16]. As pointed out by Alexander [27], a reliable experimental determination of the fractal dimension of rough surfaces is often prevented by the fact that the structures are not scale-invariant, but instead self-affine. This feature makes it impossible to determine the fractal dimension above a crossover length scale, which in the case of real physical systems is usually of the order of the smallest intrinsic length scale [28].

We show that the fronts of diffusion generated by nonequilibrium fluctuations during a diffusion process have a selfaffine fractal structure characterized by a Hurst exponent $H=1 / 2$. The local Hausdorff dimension of these fractional
Brownian surfaces is $D_{l}=3-1 / 2$, and their global fractal dimension is $D_{g}=2$. We show that the transition between the local and global regimes occurs at a crossover length of the order of the size of the molecules, so that the local region is physically not accessible. Therefore, although the fronts of diffusion are rough, they are globally flat and they do not exhibit a fractal structure. This feature of the nonequilibrium fluctuations suggests why they have never been detected during interferometric measurements aimed at recording the concentration profile by looking in the direction perpendicular to the concentration gradient (see, for example, [29]).

\section{MATHEMATICAL MODEL}

We consider a horizontal layer of thickness $L$ of a binary liquid mixture undergoing a diffusion process in the absence of gravity. We assume that the diffusive process is driven by a stationary concentration gradient $\nabla c_{0}$ aligned with the $z$ axis, and that $\nabla c_{0}$ is small with respect to the average concentration $\overline{c_{0}}, \nabla c_{0} / \overline{c_{0}} L \ll 1$. Here the concentration $c$ is the weight fraction of the denser component of the mixture. For example, a stationary concentration gradient can be obtained through the Soret effect [30]. The imposition of a constant temperature difference $\Delta T$ to the layer of liquid gives rise to a Soret mass flux $j=-\rho D S_{t} c(1-c) \nabla T$, where $\rho$ is the average density of the mixture, $S_{t}$ is the Soret coefficient, and $D$ is the mass diffusion coefficient. At steady state, the Soret flux is balanced by the diffusive flow, and a stationary concentration gradient $\nabla c=-S_{t} c(1-c) \nabla T$ is present inside the mixture. Although a stationary concentration gradient can be generated by taking advantage of the Soret effect, the presence of a temperature gradient would generate nonequilibrium temperature fluctuations, too [6]. The contribution of these fluctuations decouples from that of concentration fluctuations under the assumption that the diffusion coefficient is much smaller than the thermal diffusivity of the sample [13]. Another important case is that of isothermal diffusion, where temperature nonequilibrium fluctuations can be neglected. In the following, we will assume that diffusion occurs in one of these two regimes.

The fluid is macroscopically at rest, $\langle\vec{u}(x, y, z, t)\rangle_{t}=0$, where $u$ is the time-averaged local velocity field of the liquid. The time evolution of the concentration $c(\vec{x}, t)$ is given by advection and diffusion:

$$
\frac{\partial}{\partial t} c(\vec{x}, t)=-\vec{u}(\vec{x}, t) \cdot \vec{\nabla} c(\vec{x}, t)+D \nabla^{2} c(\vec{x}, t) .
$$

We assume that the fluctuations do not significantly alter the macroscopic concentration profile:

$$
c(\vec{x}, t)=\overline{c_{0}}+z \nabla c_{0}+\delta c(\vec{x}, t),
$$

where $\delta c(\vec{x}, t)$ represents the (small) fluctuation around the macroscopic value, $\overline{c_{0}}$ is the average macroscopic concentration, and $\nabla c_{0}$ is the uniform and constant concentration gradient. The linearization of Eq. (1) in Fourier space yields

$$
-i \omega \delta c(\vec{q}, \omega)=-\vec{u}(\vec{q}, \omega) \cdot \hat{z} \nabla c_{0}-q^{2} D \delta c(\vec{q}, \omega),
$$

where $\hat{z}$ is the unit vector pointing along the $z$ axis, directed as the concentration gradient. By solving with respect to the 
concentration, we obtain the correlation functions:

$$
\left\langle\delta c(\vec{q}, \omega) \delta c^{*}\left(\vec{q}^{\prime}, \omega^{\prime}\right)\right\rangle=\nabla c_{0}^{2} \frac{\left\langle\vec{u}(\vec{q}, \omega) \cdot \hat{z} \vec{u}^{*}\left(\vec{q}^{\prime}, \omega^{\prime}\right) \cdot \hat{z}\right\rangle}{\omega^{2}+D^{2} q^{4}} .
$$

The velocity correlation function for a liquid is [1]

$$
\begin{aligned}
& \left\langle\vec{u}(\vec{q}, \omega) \cdot \hat{z} \vec{u}^{*}\left(\vec{q}^{\prime}, \omega^{\prime}\right) \cdot \hat{z}\right\rangle \\
& \quad=\delta\left(\vec{q}-\vec{q}^{\prime}\right) \delta\left(\omega-\omega^{\prime}\right) \frac{k_{B} T v}{8 \pi^{4} \rho} \frac{q^{2}-(\vec{q} \cdot \hat{z})^{2}}{\omega^{2}+v^{2} q^{4}},
\end{aligned}
$$

where $k_{B}$ is Boltzmann's constant, $T$ is the absolute temperature, and $v$ is the kinematic viscosity.

We thus get $[10,18,31]$

$$
\begin{aligned}
\left\langle\delta c(\vec{q}, \omega) \delta c^{*}\left(\vec{q}^{\prime}, \omega^{\prime}\right)\right\rangle= & \delta\left(\vec{q}-\vec{q}^{\prime}\right) \delta\left(\omega-\omega^{\prime}\right) \frac{k_{B} T v}{8 \pi^{4} \rho} \nabla c_{0}^{2} \\
& \times \frac{q^{2}-(\vec{q} \cdot \hat{z})^{2}}{\left(\omega^{2}+D^{2} q^{4}\right)\left(\omega^{2}+v^{2} q^{4}\right)} .
\end{aligned}
$$

The static power spectrum of the fluctuations can be derived by integrating over $\omega$. We assume that the diffusion time is much longer than the viscous time, since for most liquid mixtures [10] $D \ll v$,

$$
\left\langle\delta c(\vec{q}, t) \delta c^{*}\left(\vec{q}^{\prime}, t\right)\right\rangle=\delta\left(\vec{q}-\vec{q}^{\prime}\right) \frac{k_{B} T}{8 \pi^{3} \rho} \nabla c_{0}^{2} \frac{1}{v D} \frac{1}{q^{4}} \frac{q^{2}-(\vec{q} \cdot \hat{z})^{2}}{q^{2}} .
$$

This expression shows the well-known $q^{-4}$ dependence of the power spectrum, as well as its dependence from $\nabla c_{0}^{2}[1,6]$. It is worth noting that Eq. (7) fully defines the statistical properties of the fluctuations, since they are a Gaussian random field, and thus all the correlation functions of any order can be calculated by using the Isserlis-Wick theorem [32,33].

\section{ROOT-MEAN-SQUARE AMPLITUDE OF CONCENTRATION FLUCTUATIONS}

In this section, we calculate the root-mean-square value of the fluctuations of concentration across the wave-vector range. By using polar coordinates,

$$
\begin{aligned}
\delta c_{\mathrm{rms}}^{2}= & \frac{k_{B} T}{8 \pi^{3} \rho} \nabla c_{0}^{2} \frac{1}{v D} \int_{0}^{2 \pi} d \varphi \int_{0}^{\pi} \sin (\theta) d \theta \\
& \times \int_{Q}^{\infty} q^{2} d q \frac{\sin ^{2}(\theta)}{q^{4}}=\frac{k_{B} T}{3 \pi^{2} \rho} \nabla c_{0}^{2} \frac{1}{v D} \frac{1}{Q},
\end{aligned}
$$

where $Q$ is a low-frequency cutoff. When the cutoff tends to 0 , the root-mean-square amplitude of the fluctuations diverges; this is expected, due to the $q^{-4}$ dependence of the power spectrum. However, under real conditions the fluctuations below a given $Q$ are actually quenched, leading to a finite value of the root mean square of the fluctuations. The introduction of a cutoff wave vector has many similarities with the finite-size effect determined by realistic boundary conditions. A formal calculation including the effect of boundaries is provided in Ref. [34] to evaluate the Casimir pressure determined by nonequilibrium concentration fluctuations. Remarkably, the results are quite similar despite the simple approximation introduced here by the cutoff length $Q$.
A first quenching mechanism is represented by the confinement of the fluid in the cell along the direction of the concentration gradient. Such a confinement is necessarily present in the case of concentration gradients generated by the Soret effect. In this case, both theoretical models [13] and experiments performed under microgravity conditions $[14,16]$ have shown that the quenching of the fluctuations occurs at wave vectors smaller than $Q \approx \pi / L$. Recently, the effect of confinement has been shown to affect the dynamics of the fluctuations on Earth, too [17]. A second cutoff mechanism is determined on Earth by the gravity force $[7,8]$.

Equation (8) can be rewritten in terms of the characteristic length scales of the system. To achieve this task, we introduce the microscopic length scale $a$ defined by

$$
a=\frac{k_{B} T}{6 \pi \eta D} .
$$

Physically, $a$ is of the order of the size of the molecules undergoing diffusion. In the case of spherical colloids, $D$ is the Stokes-Einstein diffusion coefficient and $a$ corresponds exactly to the radius of the particles [35]. We can thus rewrite the root-mean-square value of the concentration fluctuations as a function of the microscopic and macroscopic length scales of the system:

$$
\delta c_{\mathrm{rms}}=\frac{\nabla c_{0}}{\sqrt{3} \pi} \sqrt{a L}=\frac{\nabla c_{0}}{\sqrt{3} \pi} l .
$$

This equation shows that the mean-square amplitude of the fluctuations is proportional to the mesoscopic length scale $l$ determined by the geometric average between $a$, the molecular radius, and $L$, the thickness of the slab of liquid across which diffusion is taking place [23]. As we will see in the following, this mesoscopic length scale affects several other properties of the system. For example, $l$ determines the transition from equilibrium to nonequilibrium fluctuations in the presence of a constant concentration difference at the boundaries of the sample, and it represents the characteristic corrugation length of the diffusion wavefronts [see Eq. (32)].

Equation (10) can be rewritten as [23]

$$
\delta c_{\mathrm{rms}}=\frac{\Delta c_{0}}{\sqrt{3} \pi} \sqrt{\frac{a}{L}}
$$

where $\Delta c_{0}$ is the concentration difference across the thickness $L$ of the layer. We thus see that the fluctuation amplitude is smaller than the concentration difference $\Delta c_{0}$ by a factor of the order of 10000 , when $a$ and $L$ are, respectively, of the order of the atomic scale and of millimeters. Moreover, we see that the fluctuation amplitude vanishes as the length scale of the macroscopic concentration gradient increases.

\section{COMPARISON WITH THE EQUILIBRIUM FLUCTUATIONS}

Equilibrium fluctuations are always present in mixtures; they represent the shot noise due to the discrete nature of the liquids. The static power spectrum of the equilibrium concentration fluctuations is independent of the wave vector $q$ 
and is given by [6]

$$
\left\langle\delta c(\vec{q}, t) \delta c^{*}\left(\vec{q}^{\prime}, t\right)\right\rangle=\delta\left(\vec{q}-\vec{q}^{\prime}\right) \frac{1}{(2 \pi)^{3}} \frac{k_{B} T}{\rho}\left(\frac{\partial c}{\partial \mu}\right)_{p, T}
$$

where $\mu$ is the chemical potential difference per unit mass.

By comparing the mean-square amplitude of equilibrium fluctuations with Eq. (7), we find that they outweigh the nonequilibrium fluctuations at wave vectors such that

$$
q>\sqrt[4]{\left(\frac{\partial \mu}{\partial c}\right)_{p, T} \frac{\nabla c_{0}}{v D}}
$$

In the case of an ideal mixture,

$$
\mu=\mu_{0}+\frac{k_{B} T}{\rho a^{3}} \ln (c) .
$$

By recalling (9), we find that in the presence of a constant concentration difference at the boundaries of the sample, the transition is governed by the mesoscopic length scale $l$ :

$$
\begin{gathered}
q>\frac{1}{\sqrt{a L}} \sqrt[4]{6 \pi \frac{\Delta c_{0}^{2}}{\overline{c_{0}}} .} \quad \text { (15) } \begin{array}{l}
\text { of the fluctuations than the root-mean-square valu } \\
\text { coordinates, }
\end{array} \\
\Delta c_{\|}^{2}(\Delta z)=\frac{k_{B} T}{8 \pi^{3} \rho} \nabla c_{0}^{2} \frac{1}{v D} \int_{0}^{2 \pi} d \varphi \int_{0}^{\pi} \sin (\theta) d \theta \int_{0}^{\infty} q^{2} d q \frac{\sin ^{2}(\theta)}{q^{4}}[2-2 \exp (-i q \Delta z \cos \theta)], \\
\Delta c_{\perp}^{2}(\Delta x)=\frac{k_{B} T}{8 \pi^{3} \rho} \nabla c_{0}^{2} \frac{1}{v D} \int_{0}^{2 \pi} d \varphi \int_{0}^{\pi} \sin (\theta) d \theta \int_{0}^{\infty} q^{2} d q \frac{\sin ^{2}(\theta)}{q^{4}}[2-2 \exp (-i q \Delta x \sin \theta \cos \varphi)] .
\end{gathered}
$$

This condition implies that in the case of a simulation performed over a given box size $\lambda=2 \pi / q$, there is a minimum concentration gradient for observing nonequilibrium phenomena, $\nabla c_{0}>a q^{2} \sqrt{\overline{c_{0}} /(6 \pi)}$.

\section{ROOT-MEAN-SQUARE CONCENTRATION DIFFERENCE BETWEEN TWO POINTS}

In this section, we consider two points inside the liquid mixture, displaced along or perpendicular to the concentration gradient, and we evaluate the root-mean-square value of the concentration difference between them. We call the two quantities $\Delta c_{\|}(\Delta z)$ and $\Delta c_{\perp}(\Delta x)$ :

$$
\begin{aligned}
\Delta c_{\|}(\Delta z) & =\sqrt{\left\langle[\delta c(0, t)-\delta c(\hat{z} \Delta z, t)]^{2}\right\rangle} \\
\Delta c_{\perp}(\Delta x) & =\sqrt{\left\langle[\delta c(0, t)-\delta c(\hat{x} \Delta x, t)]^{2}\right\rangle}
\end{aligned}
$$

where $\hat{x}$ and $\hat{z}$ are the unit vectors perpendicular and parallel to the concentration gradient, respectively.

We will see that the integrals leading to such quantities do not diverge, hence they give a better local characterization of the fluctuations than the root-mean-square value. In polar

The first can be integrated in $\theta$ and $\varphi$ :

$$
\Delta c_{\|}^{2}(\Delta z)=2 \frac{k_{B} T}{\pi^{2} \rho} \nabla c_{0}^{2} \frac{1}{v D} \int_{0}^{\infty} d q \frac{\frac{1}{3}(q \Delta z)^{3}+q \Delta z \cos (q \Delta z)-\sin (q \Delta z)}{q^{5} \Delta z^{3}},
$$

and then in $q$,

$$
\Delta c_{\|}^{2}(\Delta z)=\frac{k_{B} T}{8 \pi \rho} \nabla c_{0}^{2} \frac{1}{v D} \Delta z .
$$

The integrals in the expression for $\Delta c_{\perp}(\Delta x)$ cannot be easily calculated explicitly. By changing the integration variable to $t=q \Delta x$, we get

$$
\Delta c_{\perp}^{2}(\Delta x)=\frac{k_{B} T}{8 \pi \rho} \nabla c_{0}^{2} \frac{1}{v D} \Delta x \Gamma^{2},
$$

where

$$
\Gamma=\frac{1}{\pi} \sqrt{\int_{0}^{2 \pi} d \varphi \int_{0}^{\pi} d \theta \int_{0}^{\infty} d t \frac{\sin ^{3}(\theta)}{t^{2}}[2-2 \exp (-i t \sin \theta \cos \varphi)] .}
$$

The integrals converge and can be easily calculated numerically; we obtain the value $\Gamma \approx 1.21$. A confirmation of this result has been obtained in a recent study on the effect of the confinement on the dynamics of nonequilibrium concentration fluctuations [17]. This study showed that the confinement effect starts to be felt at the reduced wave number $q L=$ $5.18=\mathrm{Ra}_{\mathrm{sc}}^{1 / 4}$, where $\mathrm{Ra}_{\mathrm{sc}}=720$ is the critical solutal Rayleigh number. This implies that the lateral size of a fluctuation when confinement starts to be important is $\lambda=(2 \pi / 5.18)$ $L \approx 1.2 L$.
We thus finally obtain

$$
\begin{gathered}
\Delta c_{\|}(\Delta z)=\nabla c_{0} \sqrt{\frac{k_{B} T}{8 \pi \rho} \frac{1}{\nu D} \Delta z,} \\
\Delta c_{\perp}(\Delta x)=\Gamma \nabla c_{0} \sqrt{\frac{k_{B} T}{8 \pi \rho} \frac{1}{v D} \Delta x .}
\end{gathered}
$$

We can notice that the integrals lead to the finite values expressed by Eqs. (24) and (25), without divergences and 
without the need to impose the low-frequency cutoff, at variance with the root-mean-square concentration fluctuation. This notwithstanding, in the presence of such a cutoff $Q$, we can expect that Eqs. (24) and (25) hold only for $\Delta z \ll 1 / Q$ and $\Delta x \ll 1 / Q$. In the opposite limit, $\Delta x \rightarrow+\infty$ and $\Delta z \rightarrow$ $+\infty$, we can easily calculate

$$
\lim _{\Delta x \rightarrow+\infty} \Delta c_{\perp}(\Delta x)=\lim _{\Delta z \rightarrow+\infty} \Delta c_{\|}(\Delta z)=\sqrt{2} \delta c_{\mathrm{rms}},
$$

where $\delta c_{\mathrm{rms}}$ is defined (i.e., finite) in the presence of the lowfrequency cutoff $Q$.

We can thus assume that, in the presence of the cutoff, the values of $\Delta c_{\perp}$ and $\Delta c_{\|}$increase as expressed by Eqs. (24) and (25) for $\Delta z \ll 1 / Q$ and $\Delta x \ll 1 / Q$, while they saturate to $\delta c_{\mathrm{rms}}$ at $\Delta z \gg 1 / Q$ and $\Delta x \gg 1 / Q$; the behavior around the transition, roughly at $1 / Q$, depends on the physical effect that induces the quenching of the long-wavelength fluctuations, e.g., the finite cell size.

From Eqs. (24) and (25), we can conclude that the fluctuations are slightly anisotropic, the ratio between their root-mean-square amplitude in directions perpendicular and parallel to the macroscopic concentration gradient being of the order of $\Gamma$.

We can further elaborate Eqs. (24) and (25) by introducing the microscopic length scale $a$ defined in (9):

$$
\begin{gathered}
\Delta c_{\|}(\Delta z)=\sqrt{\frac{3}{4}} \nabla c_{0} \sqrt{a \Delta z}, \\
\Delta c_{\perp}(\Delta x)=\Gamma \sqrt{\frac{3}{4}} \nabla c_{0} \sqrt{a \Delta x} .
\end{gathered}
$$

By comparing with Eq. (10), we see that the values reach the saturation $\delta c_{\text {rms }}$ approximately at $Q \approx 1 / L$.

\section{FRONTS OF DIFFUSION}

In this section, we investigate the properties of the fronts of diffusion, defined as the surfaces $z=h(x, y, t)$ where the concentration is constant, $c(x, y, h(x, y, t))=$ const.

\section{A. Corrugation}

To evaluate the corrugation of the diffusion wavefront, we consider the wavefront at average height $z=0:\langle h(x, y, t)\rangle=$ 0 ; since the concentration fluctuations are small with respect to the macroscopic concentration profile, we can approximate

$$
\begin{aligned}
c[x, y, h(x, y, t)]= & c(x, y, z=0, t) \\
& +\frac{\partial}{\partial z} c(x, y, z=0, t) h(x, y, t) .
\end{aligned}
$$

By applying Eq. (2),

$$
\begin{aligned}
c[x, y, h(x, y, t)]= & \overline{c_{0}}+\delta c(x, y, z=0, t) \\
& +\left[\nabla c_{0}+\frac{\partial}{\partial z} \delta c(x, y, z=0, t)\right] h(x, y, t) .
\end{aligned}
$$

We neglect the derivative of the concentration $\delta c$ with respect to $z$ because it is much smaller than $\nabla c_{0}$, the gradient of the macroscopic concentration profile. Moreover, we notice that $c[x, y, h(x, y, t)]$ is a constant,

$$
h(x, y, t)=\mathrm{const}-\frac{c(x, y, z=0, t)}{\nabla c_{0}} .
$$

Since $h$ is proportional to the local concentrations, by using Eq. (10) we get

$$
\Delta h_{\mathrm{rms}}=\sqrt{\frac{2}{\pi}} \sqrt{a L} .
$$

Therefore, the thickness of the corrugation of the fronts of diffusion is of the order of the mesoscopic length scale $l$.

\section{B. Self-affine structure}

We now evaluate the root-mean-square value of $\Delta h(\Delta x)$, that is, the root-mean-square variation of the displacement of the fronts of diffusion between two points spaced perpendicular to the gradient of the macroscopic concentration profile, at a distance $\Delta x$. From Eq. (27), we get

$$
\Delta h(\Delta x)=\Gamma \sqrt{\frac{3}{4}} \sqrt{a \Delta x} .
$$

Equation (32) shows that the fronts of diffusion have a self-affine fractal structure. More precisely, the fact that Eq. (32) is in the form $\Delta h(\Delta x) \propto \Delta x^{H}$ indicates that the fronts of diffusion are fractional Brownian surfaces with a Hurst exponent [36] $H=1 / 2$.

Equation (32) suggests that $h$ is not a smooth function of $x$ and $y$, since the derivative of the square root diverges in 0 . We can indeed try to calculate the root mean square of the derivative of $h$ with respect to $x$; in turn, it is related to the root mean square of the derivative of $\delta c$ with respect to $x$. The calculation can be easily performed in Fourier space,

$$
\begin{aligned}
\left(\frac{\partial \delta c}{\partial x}\right)_{\mathrm{rms}}^{2} \propto & \left(\frac{\partial h}{\partial x}\right)_{\mathrm{rms}}^{2} \propto \frac{k_{B} T}{8 \pi^{3} \rho} \nabla c_{0}^{2} \frac{1}{\nu D} \int_{0}^{2 \pi} d \varphi \int_{0}^{\pi} d \theta \\
& \times \int_{Q}^{\infty} d q \frac{\sin ^{3}(\theta)}{q^{2}}[q \sin (\theta) \cos (\varphi)]^{2}
\end{aligned}
$$

The integral has a short-wavelength divergence for $q \rightarrow+\infty$, which is thus not cured by the presence of the cutoff $Q$. This supports the conclusion that $h$ is strongly not smooth and its derivative is not bounded.

\section{Simulation of the concentration fluctuations}

To visually represent the self-affine surfaces of the fronts of diffusion, we have performed numerical simulations. We consider vertical sections of the liquid system along a plane parallel to the concentration gradient $\nabla c_{0}$. The representations are obtained in two ways: by mapping the concentrations on the plane to image gray-scale intensities, and by drawing the isoconcentration curves, representing the diffusion wavefronts.

We define $\delta c\left(q_{x}, q_{z}, t\right)$ as the Fourier transform of the concentration on the $(x, z)$ plane, at $y=0$; it is connected to the three-dimensional Fourier transform $\delta c\left(q_{x}, q_{y}, q_{z}, t\right)$ :

$$
\delta c\left(q_{x}, q_{z}, t\right)=\int \delta c\left(q_{x}, q_{y}, q_{z}, t\right) d q_{y} .
$$


The power spectrum of the concentration fluctuations on a vertical section can thus be obtained from Eq. (7):

$$
\begin{aligned}
\left\langle\delta c\left(q_{x}, q_{z}, t\right) \delta c^{*}\left(q_{x}^{\prime}, q_{z}^{\prime}, t\right)\right\rangle= & \delta\left(q_{x}-q_{x}^{\prime}\right) \delta\left(q_{z}-q_{z}^{\prime}\right) \frac{k_{B} T}{8 \pi^{3} \rho} \nabla c_{0}^{2} \\
& \times \frac{1}{v D} \int \frac{q_{x}^{2}+q_{y}^{2}}{\left(q_{x}^{2}+q_{y}^{2}+q_{z}^{2}\right)^{3}} d q_{y} .
\end{aligned}
$$

The integral can be easily computed as follows:

$$
\begin{aligned}
& \left\langle\delta c\left(q_{x}, q_{z}, t\right) \delta c^{*}\left(q_{x}^{\prime}, q_{z}^{\prime}, t\right)\right\rangle \\
& \quad=\delta\left(q_{x}-q_{x}^{\prime}\right) \delta\left(q_{z}-q_{z}^{\prime}\right) \frac{k_{B} T}{8 \pi^{2} \rho} \nabla c_{0}^{2} \frac{1}{v D} \frac{4 q_{x}^{2}+q_{z}^{2}}{8{\sqrt{q_{x}^{2}+q_{z}^{2}}}^{5}} .
\end{aligned}
$$

We used this expression for generating the Fourier transforms of the images of the fluctuations, reported in Fig. 1. The simulation involves all the length scales down to the size of the pixel, i.e., the fluid is considered as continuous across all the length scales that are represented in the picture. However, the molecular size enters in the calculation through Eq. (9). We notice that the fluctuations disappear as the slab thickness increases with respect to the microscopic scale.

\section{Fractal dimension}

From Eq. (7), one can appreciate that the fluctuations can be rescaled: if we zoom the spatial scales by a factor $\lambda$, the amplitude of fluctuation decreases by a factor $\lambda^{2}$. It is worth noting that this is not a physical scaling, because only the fluctuations are rescaled by this factor, while the macroscopic concentration profile is not; for example, if the profile is linear, it is decreased by a factor $\lambda$.

A similar argument can be used, together with Eq. (30), to show that the corrugation of the isoconcentration surfaces decreases by a factor $\sqrt{\lambda}$ when the spatial scales are zoomed by a factor $\lambda$. Such scaling laws, together with (32), show that the fronts of diffusion have a self-affine fractal structure and are not scale-invariant, due to the anisotropy of the system determined by the external gradient. The determination of the fractal dimension of self-affine surfaces has been widely debated in the past (see, for example, [28] and references therein). The lack of self-similarity and the presence of spatial anisotropy give rise to fractal structures whose fractal dimension is scale-dependent. Since the fronts of diffusion have a self-affine structure corresponding to a Brownian surface [37], we discuss below in detail the determination of the fractal dimension for this kind of system.

The isoconcentration curves shown in Fig. 1 are sections of the isoconcentration surfaces for $y=0$. From Eq. (32), we see that the root-mean-square variation of the height $h$ depends as a square root on the displacement. The isoconcentration curve can thus be interpreted as the graph of a Wiener process, and $h$ as a Brownian motion (a random walk) as a function of $x$. Analogously, the isoconcentration surfaces can be defined as Brownian surfaces.

In general, the fractal dimension $D$ of the graph of a function is connected to the power-law exponent of the
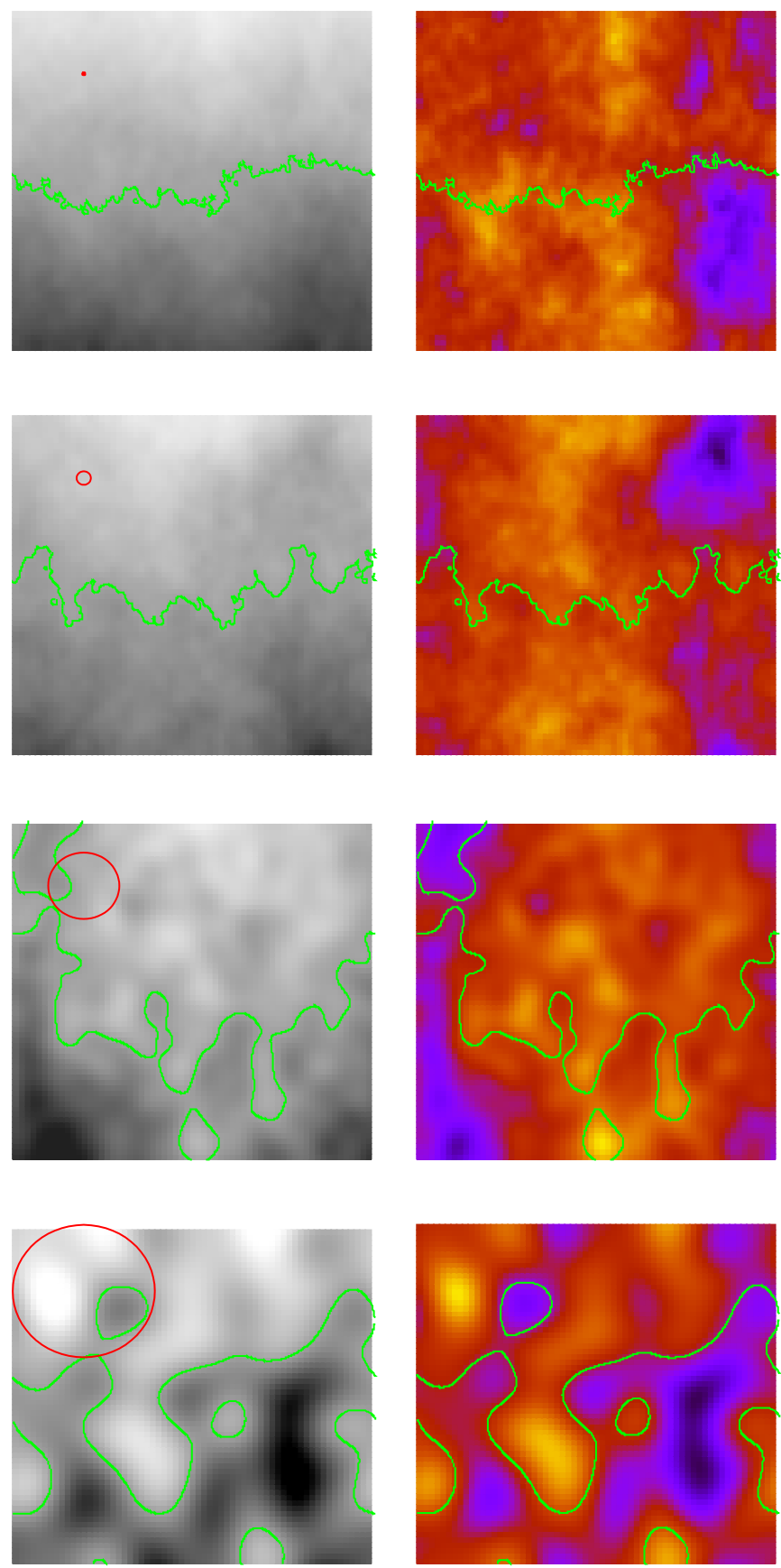

FIG. 1. Vertical section of the fluid slab where diffusion takes place. The four samples are simulated with different slab thickness. For each sample, the left panel maps the concentration profile, represented in gray scale, while the right panel maps variations of concentration with respect to the constant-gradient macroscopic concentration profile. Isoconcentration curves, i.e., the diffusion wavefronts, are also shown. The circle represents the microscopic length scale.

correlation function, which turns out to be [37] $4-2 D$; in the case of the Brownian functions, the exponent is $1 / 2$ [see Eq. (32)], leading to a fractal dimension $D=1.5$ for the isoconcentration curves. An analogous consideration would lead to a fractal dimension $D=2.5$ for the isoconcentration surfaces. 


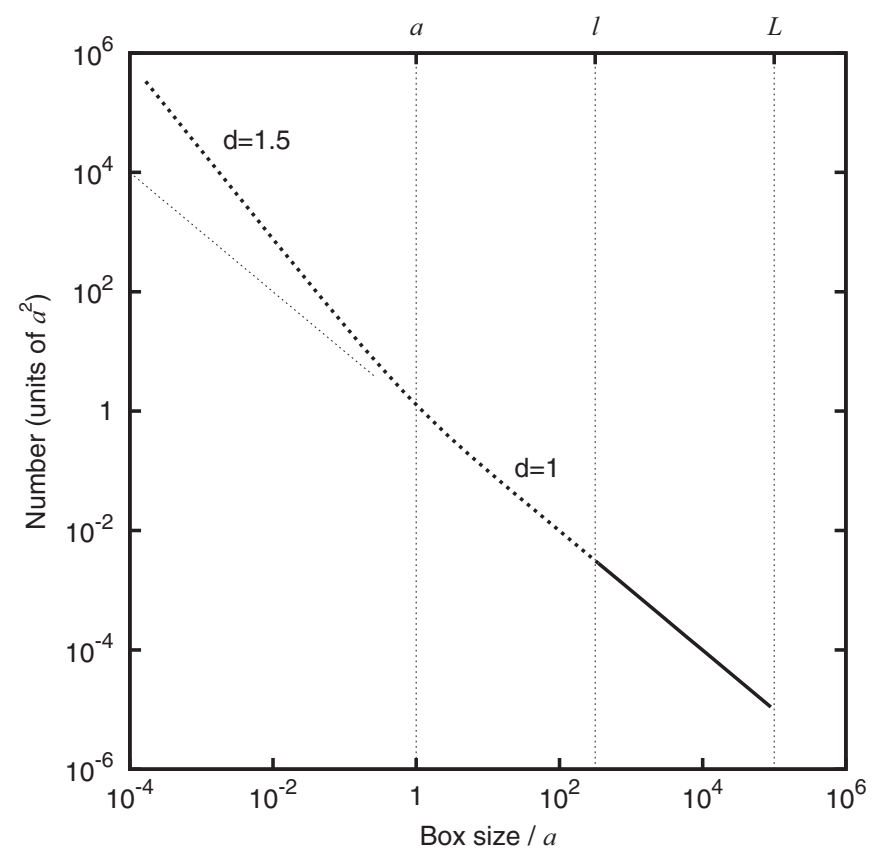

FIG. 2. Box-counting method applied to the isoconcentration curves. The graph represents the count, normalized with respect to a square system of size $a$ (the microscopic length scale).

In practice, the evaluation of the fractal dimension of the graph of a Brownian function is known to be problematic. First of all, the box-counting method gives a local fractal dimension $D_{l}=d-H=1.5$ [36], where $d$ is the dimensionality of the space. However, this result is obtained only in the limit of spatial scales $r \rightarrow 0$, while on a global scale in the limit $r \rightarrow$ $+\infty$, one obtains the global fractal dimension $D_{g}=1$ [38]. The transition between the two behaviors takes place at the crossover scale defined by the relation $\Delta h\left(\Delta x_{c}\right) \approx \Delta x_{c}$. In practice, for real experimental systems, the crossover length scale $\Delta x_{c}$ coincides with the smallest nontrivial length scale, and the fractal structure is not expected to be apparent in experiments [27,28]. According to Eq. (32), we see that in the case of the fronts of diffusion generated by nonequilibrium fluctuations in liquid mixtures, the transition is approximately at the microscopic length scale $a$.

In Fig. 2, we report the result of the box-counting method applied to the isoconcentration curves, calculated in the limit of vanishing concentration gradient. We see that the fractal dimension is compatible with $d=1.5$ in the limit of small box size, but it becomes $d=1$ for large box size, as for Brownian functions; one can appreciate that the transition is approximately at the molecular radius $a$.

We can thus conclude that the isoconcentration curves (surfaces) have a Hausdorff dimension $d=1(d=2)$ and do not exhibit a fractal structure. An extrapolation down to scales smaller than the molecular radius would lead to fractal objects, but this is strictly a theoretical result connected to the self-affine structure of the equations, without any physical counterpart in a real system.

\section{CONCLUSIONS}

We have considered the spatial correlation properties of concentration nonequilibrium fluctuations parallel and perpendicular to the macroscopic gradient, and we showed that the fluctuations are anisotropic with an aspect ratio $\Gamma \approx 1.2$. We have investigated the structure of the fronts of diffusion, and we showed that they exhibit the self-affine structure of a Brownian surface. The local fractal dimension of this surface should be $D_{l}=2.5$, and the global fractal dimension should be $D_{g}=2.0$. However, the transition between the two regimes occurs at a crossover length scale of the order of the microscopic length scale of the system. In principle, by using complex liquids, such as a colloidal suspension [39], or supraparticles composed of many particles such as micelles or vesicles [40], the microscopic length scale $a$ can become accessible by using light-scattering methods. Indeed, under these circumstances, $a$ would correspond to the size of the colloidal particles. However, probing length scales smaller than $a$ would provide access to the inner particles, and no fractal structure would be apparent (unless the particles have an internal fractal structure themselves, such as colloidal aggregates or polymers). This is because the impossibility of detecting a fractal structure of the diffusion fronts is not due to the lack of resolution of the experimental techniques used to investigate the system. Rather, it is an intrinsic limitation of the investigated system. Therefore, the fractal local structure is not apparent in experiments and simulations, and the fronts of diffusion, although corrugated, are globally flat.

\section{ACKNOWLEDGMENTS}

We thank A. Donev for preliminary discussion on the fractal structure of the fronts of diffusion. F.C. acknowledges support from the Centre National d'Etudes Spatiales (CNES).
[1] B. M. Law and J. C. Nieuwoudt, Phys. Rev. A 40, 3880 (1989).

[2] J. C. Nieuwoudt and B. M. Law, Phys. Rev. A 42, 2003 (1990).

[3] G. Grinstein, in Scale Invariance, Interfaces, and NonEquilibrium Dynamics, edited by A. E. A. Mckane (Plenum, New York, 2006), pp. 261-293.

[4] W. B. Li, P. N. Segré, R. W. Gammon, and J. V. Sengers, Physica A 204, 399 (1994).

[5] W. B. Li, P. N. Segré, R. W. Gammon, and J. V. Sengers, J. Phys.: Condens. Matter 6, A119 (1994).
[6] J. M. Ortiz de Zárate and J. V. Sengers, Hydrodynamic Fluctuations in Fluids and Fluid Mixtures (Elsevier, Amsterdam, 2006).

[7] P. N. Segré and J. V. Sengers, Physica A 198, 46 (1993).

[8] A. Vailati and M. Giglio, Phys. Rev. Lett. 77, 1484 (1996).

[9] A. Vailati and M. Giglio, Nature (London) 390, 262 (1997).

[10] A. Vailati and M. Giglio, Phys. Rev. E 58, 4361 (1998).

[11] D. Brogioli, A. Vailati, and M. Giglio, Phys. Rev. E 61, R1 (2000). 
[12] F. Croccolo, D. Brogioli, A. Vailati, M. Giglio, and D. S. Cannell, Phys. Rev. E 76, 041112 (2007).

[13] J. M. Ortiz de Zárate, F. Peluso, and J. V. Sengers, Eur. Phys. J. E 15, 319 (2004).

[14] A. Vailati, R. Cerbino, S. Mazzoni, C. J. Takacs, D. S. Cannell, and M. Giglio, Nat. Commun. 2, 290 (2011).

[15] R. Cerbino, Y. Sun, A. Donev, and A. Vailati, Sci. Rep. 5, 14486 (2015).

[16] F. Croccolo, C. Giraudet, H. Bataller, R. Cerbino, and A. Vailati, Micrograv. Sci. Technol. 28, 467 (2016).

[17] C. Giraudet, H. Bataller, Y. Sun, A. Donev, J. M. Ortiz de Zárate, and F. Croccolo, Europhys. Lett. 111, 60013 (2015).

[18] D. Brogioli and A. Vailati, Phys. Rev. E 63, 012105 (2000).

[19] A. Donev, J. B. Bell, A. de la Fuente, and A. L. Garcia, Phys. Rev. Lett. 106, 204501 (2011).

[20] A. Donev, T. G. Fai, and E. Vanden-Eijnden, J. Stat. Mech. (2014) P04004.

[21] T. R. Kirkpatrick, J. M. Ortiz de Zárate, and J. V. Sengers, Phys. Rev. Lett. 115, 035901 (2015).

[22] T. R. Kirkpatrick, J. M. Ortiz de Zárate, and J. V. Sengers, Phys. Rev. E 93, 012148 (2016).

[23] T. R. Kirkpatrick, J. M. Ortiz de Zárate, and J. V. Sengers, Phys. Rev. E 93, 032117 (2016).

[24] A. K. Bhattacharjee, K. Balakrishnan, A. L. Garcia, J. B. Bell, and A. Donev, J. Chem. Phys. 142, 224107 (2015).

[25] D. Bedeaux, J. M. Ortiz de Zárate, I. Pagonabarraga, J. V. Sengers, and S. Kjelstrup, J. Chem. Phys. 135, 124516 (2011).
[26] A. Lesne and M. Lagues, Scale Invariance (Springer, Berlin, 2012).

[27] S. Alexander, in Transport and Relaxation in Random Materials, edited by J. R. Klafter, J. Rubin, and M. F. Shlesinger (World Scientific, Singapore, 1986), pp. 59-71.

[28] Dynamics of Fractal Surfaces, edited by F. Family and T. Vicsek (World Scientific, Singapore, 1991).

[29] D. H. J. F. Torres, A. Komiya and S. Maruyama, J. Chem. Phys. 139, 074203 (2013).

[30] S. R. de Groot and P. Mazur, Nonequilibrium Thermodynamics (North-Holland, Amsterdam, 1962).

[31] L. D. Landau and E. M. Lifshitz, Fluid Mechanics (Pergamon, New York, 1959).

[32] L. Isserlis, Biometrika 12, 134 (1918).

[33] G. C. Wick, Phys. Rev. 80, 268 (1950).

[34] J. M. Ortiz de Zárate, T. R. Kirkpatrick, and J. V. Sengers, Eur. Phys. J. E 38, 99 (2015).

[35] C. Tanford, Physical Chemistry of Macromolecules (Wiley, New York, 1961).

[36] B. B. Mandelbrot, Phys. Scr. 32, 257 (1985).

[37] K. Falconer, Fractal Geometry: Mathematical Foundations and Applications, 2nd ed. (Wiley, New Jersey, 2003).

[38] H. Takayasu, Fractals in the Physical Sciences (Manchester University Press, Manchester, 1990).

[39] F. Giavazzi, G. Savorana, A. Vailati, and R. Cerbino, Soft Matter 12, 6588 (2016).

[40] L. Golubović and M. Golubović, Phys. Rev. E 56, 3219 (1997). 